\title{
Las representaciones sociales de la clasificación de escuelas presentes en los discursos en medios de comunicación escritos
}

\author{
The social representations of classification of schools present \\ in the discourse of print media
Representações sociais sobre a classificação das escolas apresentam nos discursos mídia impressa

\author{
Olivares-Donoso, Ruby ${ }^{a}$ \\ aPontificia Universidad Católica, Santiago, Chile. \\ Correo electrónico: rrolivares@uc.cl
}

\begin{abstract}
RESUMEN
El 11 de agosto de 2011 se promulgó la Ley de Aseguramiento de la Calidad de la Educación Escolar (Ley $\mathrm{N}^{\circ}$ 20.529), la que, entre otros aspectos, creó la Agencia de Calidad de la Educación, una de cuyas funciones es ordenar los establecimientos según los resultados de aprendizaje y otros indicadores de calidad educativa. Esta función ha generado un intenso debate, a favor y en contra de la propuesta, en los medios de comunicación escritos. El objetivo del presente trabajo es conocer las representaciones sociales de la clasificación de escuelas presentes en el discurso de distintos actores del sistema educativo, para ello se analizaron 24 documentos utilizando la metodología de la Teoría Fundamentada. Los resultados indican que los actores del sistema educativo tienen representaciones sociales diferenciadas acerca de la clasificación de escuelas dependiendo de la posición, a favor o en contra, que adopten frente a este tema.
\end{abstract}

Palabras clave: clasificación de escuelas, representaciones sociales, discurso, medios de comunicación.

\begin{abstract}
August 11th, 2011, the Law of Education Quality Assurance (Law N 20.529) was enacted, which, among other things, created the Agency for Education Quality Assurance, one of whose functions is to sort schools according to learning results and other indicators of educational quality. This function has generated intense debate, both for and against the proposal, through the print media. The aim of this study is to know the social representations of the classification of schools present in the discourse of different actors in the education system, for which 24 documents were analyzed using the methodology of Grounded Theory. The results indicate that the actors of the educational system have distinct social representations about the classification of schools depending on the position, for or against, taken in regard to this issue.
\end{abstract}

Key words: classification of schools, social representations, discourse, print media.

\section{RESUMO}

No dia 11 de agosto de 2011, promulgou-se a Lei de Garantia de Qualidade da Educação Escolar (Lei $\mathrm{N}^{\circ}$ 20.529) que, entre outras coisas, criou a Agência de Qualidade da Educação que, entre outras, tem a função de classificar as escolas de acordo com resultados da aprendizagem e outros indicadores da qualidade educativa. A referida função tem gerado um debate intenso nos meios de comunicação impressos, seja ele favorável à proposta ou contra ela. Com objetivo de compreender as representações sociais da classificação das escolas presentes no discurso de diferentes atores no sistema educativo, analisaram-se 24 documentos, utilizando-se a metodologia da Teoria Fundamentada nos Dados. Resultados indicam que os atores do sistema educacional têm representações sociais distintas acerca da classificação das escolas dependendo da posição tomada em relação ao tema, favorável a ele ou contra ele.

Palavras chave: classificação das escolas, representações sociais, discurso, meios de comunicação. 


\section{INTRODUCCIÓN}

En países como Inglaterra, Noruega y Francia, los gobiernos publican los datos de desempeño obtenidos por las escuelas en pruebas estandarizas aplicadas a nivel nacional (e.g. Elstead, 2009; Karsten, Visscher \& de Jong, 2001; West \& Pennell, 2000). Un caso emblemático corresponde a Inglaterra, que en 1988 promulgó la Ley de Reforma de la Educación, la que estaba orientada a "aumentar la elección de los padres y de ese modo mejorar la rendición de cuentas de las escuelas financiadas por el Estado" (Machin \& Vignoles, 2006: 3). La idea era que los padres pudieran elegir, a partir de la información disponible, a qué escuela querían que asistiera su hijo. En 1992 el gobierno publicó las primeras tablas de desempeño para la educación secundaria y, en 1996 para la primaria, estas fueron construidas con los puntajes promedio por escuela (Dearden \& Vignoles, 2011). Sin embargo, ante las críticas recibidas por el uso de datos "brutos", el gobierno desarrolló, primero, tablas de valor agregado, las que miden el desempeño de las escuelas en función de la ganancia promedio de sus alumnos y, después, tablas de valor agregado contextualizado, estas toman en cuenta variables de contexto del estudiante (Dearden \& Vignoles, 2011). Estos datos son utilizados por los medios de comunicación escrita para construir "tablas de clasificación", las que han generado varias críticas, principalmente las que utilizan datos "brutos", porque centran la atención de la opinión pública en las "mejores" y las "peores" escuelas (e.g. Power \& Frandji, 2010; Elstead, 2009). En este sentido, las investigaciones demuestran que la publicación de tablas de clasificación con datos "brutos" produce efectos secundarios negativos, como la estigmatización de las escuelas y la desmotivación tanto de profesores como de estudiantes (e.g. Levačić \& Woods, 2002; Woods \& Levačić, 2002).

En Chile se está trabajando en el diseño de una metodología que clasificará a las escuelas, la que debe ser aprobada por decreto supremo del Ministerio de Educación. De acuerdo con la Agencia de Calidad, esta clasificación "no constituye un ranking de establecimientos educacionales, porque su énfasis está en identificar cuatro grandes grupos de establecimientos de acuerdo con su desempeño, sin hacer distinciones entre los establecimientos al interior de cada categoría, porque no es ese su objetivo" (2013a: 11). Sin embargo, considerando la evidencia internacional, estos datos podrían ser utilizados por los medios de comunicación escrita para construir sus propios rankings -tablas de clasificación-, práctica que ya se realiza con los puntajes obtenidos por las escuelas en el SIMCE (e.g. El Mercurio, 2013). En este sentido, resulta interesante estudiar, debido al carácter masivo que tienen los medios de comunicación escrita, las representaciones sociales que se transmiten en ellos, aun antes de que esta política sea aplicada.

\section{MARCO TEÓRICO Y CONCEPTUAL}

\subsection{REPRESENTACIONES SOCIALES}

El concepto clave para abordar el problema planteado es el de "representaciones sociales". Este concepto fue desarrollado por Moscovici (1979), basado en el concepto de "representaciones colectivas" de Durkheim (2001), quien las diferenció de las "representaciones individuales" a partir "del supuesto de que las representaciones individuales existían pero 
no eran adecuadas para el trabajo del científico social, ya que eran altamente subjetivas, heterogéneas y permanentemente cambiantes" (Farr, 2003: 153), impulsando así la separación entre sociología y psicología. En este sentido, Moscovici consideró que el adjetivo de "sociales" calificaba a las representaciones de una manera más adecuada que el de "colectivas" (Farr, 2003) y así definió las representaciones sociales como:

Sistemas de valores, ideas y prácticas que tienen una doble función: en primer lugar, establecer un orden que permita a los individuos orientarse en su mundo social y material y dominarlo; y, en segundo término, permitir la comunicación entre los miembros de una comunidad, aportándoles un código para el intercambio social y un código para denominar y clasificar de manera inequívoca los distintos aspectos de su mundo y de su historia individual y grupal (Cit. en Duveen y Lloyd, 2003: 29-30).

Jodelet (1986), uno los autores seguidores de la teoría de las representaciones sociales de Moscovici plantea que estas se presentan de distintas formas, como imágenes, sistemas de referencia, categorías, teorías, y en algunos casos pueden ser todas ellas; sin embargo, independiente de su forma la representación social siempre se trata de "una manera de interpretar y de pensar nuestra realidad cotidiana, una forma de conocimiento social (énfasis de la autora)" (473). Además, Jodelet (1986) establece que:

el concepto de representación social designa una forma de conocimiento específico, el saber de sentido común, (...) una forma de pensamiento social.

Las representaciones sociales constituyen modalidades de pensamiento práctico orientados hacia la comunicación, la comprensión y el dominio del entorno social, material e ideal (énfasis de la autora) (474).

A partir de lo anterior, Jodelet (1986) plantea que la representación social se define por un contenido, este se relaciona con un objeto, y corresponde a la representación social de un sujeto respecto de otro sujeto. De esta forma, "toda representación social es representación de algo y de alguien” (Jodelet, 1986: 475). En este sentido, las representaciones sociales son construcciones simbólicas, las cuales no reemplazan al objeto representado en la mente de los individuos, sino que en ellas quedan plasmados los aspectos sociales, culturales e históricos (Petracci y Kornblit, 2004). Además, de acuerdo con Petracci y Kornblit (2004), las representaciones sociales "son construidas a partir de los procesos de interacción y comunicación social, las conversaciones de la vida diaria, la recepción de los medios masivos -todos ellos procesos comunicacionales a los que también reconstruyen- y se cristalizan en prácticas sociales" (92).

Para Abric (2001), otro autor seguidor de la teoría de Moscovici, la representación social está constituida por "un conjunto de informaciones, de creencias, de opiniones y de actitudes a propósito de un objeto dado (...) [y] este conjunto de elementos es organizado y estructurado" (18). Así, la representación social corresponde a un punto de vista del mundo que permite, tanto al individuo como al grupo, dar sentido a sus conductas y así entender la realidad mediante su propio sistema de referencias. En este sentido, Abric (2001) plantea que la representación social "funciona como un sistema de interpretación de la realidad que rige las relaciones de los individuos con su entorno físico y social, 
ya que determinará sus comportamientos o sus prácticas. Es una guía para la acción (énfasis del autor), orienta las acciones y las relaciones sociales" (13).

Los procesos en donde se produce la elaboración de las representaciones sociales corresponden a la objetivación y el anclaje (Jodelet, 1986). La objetivación se define como una operación que da imagen y estructura, ya que concretiza lo abstracto (Jodelet, 1986). Para Moscovici, este proceso "es el verdadero núcleo de la representación social" (Cit. en Petracci y Kornblit, 2004: 92). Consta de tres fases: a) construcción selectiva, proceso por el que los individuos seleccionan las informaciones que circulan respecto de una teoría, y se apropian de ellas al separarlas del campo científico al que pertenecen y del grupo de expertos que las concibió; b) esquematización estructurante, se refiere al proceso donde los diferentes elementos seleccionados y apropiados en la fase anterior son organizados en una imagen del objeto que representará un "modelo figurativo" o "núcleo figurativo"; y c) naturalización, proceso por el cual el "modelo figurativo" construido en la fase previa adquiere un estatus de evidencia (Jodelet, 1986). Además, el anclaje se define como "el enraizamiento social de la representación social y de su objeto" (Jodelet, 1986: 486), de tal forma que los elementos objetivados se integran a nuestros esquemas de pensamiento (Petracci y Kornblit, 2004).

Moscovici (1979) plantea que, en un nivel superficial, las representaciones sociales constituyen universos de opiniones -específicos para cada grupo social- los cuales tienen tres dimensiones:

a) Actitud: hace referencia a la disposición general, positiva o negativa, de las personas respecto del objeto o situación social representada. En este sentido, esta dimensión tiene que ver con lo afectivo.

b) Información: corresponde a la forma en la que se organizan los conocimientos y nociones que un grupo social tiene en relación con el objeto o situación social. La cantidad y calidad de la información que maneja una persona está definida por su pertenencia a un determinado grupo social.

c) Campo de representación: se relaciona con el contenido concreto de la representación, aquí es donde se materializa la representación, por medios de una imagen o modelo del objeto o situación social representada.

Estas dimensiones dan cuenta del contenido y del sentido de la representación social (Moscovici, 1979), por lo que para conocer una representación social es necesario establecer qué se sabe (información), cómo se interpreta (campo de representación) y cómo se actúa (actitud) (Araya, 2002).

Por su parte, Abric (2001) plantea que las representaciones sociales responden a cuatro funciones:

1. Cognitivas (de saber): permiten entender y explicar la realidad. El sentido común constituye un saber práctico que permite asimilar comprensivamente el contexto del que forman parte los individuos. Además, es la condición necesaria para comunicarnos con otros, y para transmitir y difundir ese saber.

2. Identitarias: definen y otorgan identidad al sujeto y a su grupo. El sentido de pertenencia y la identificación con un colectivo aluden a la necesidad de los sujetos de ubicarse en un referente valórico y normativo que los diferencie de otros. Las 
representaciones, en este sentido, resguardan la imagen positiva del grupo y cumplen un control social en relación con los procesos de socialización.

3. De orientación: conducen las conductas y las prácticas. En este sentido, son juicios de anticipación que determinan expectativas. Las representaciones que un sujeto posea de un determinado objeto, condicionan su comportamiento y crean un tipo de relación característica del sujeto con ese objeto. Además, son prescriptivas de comportamientos y prácticas sociales.

4. Justificadoras: permiten explicar y justificar a posteriori las posturas y las conductas adoptadas. De esta forma, refuerzan la posición de un grupo, manteniendo y justificando la diferenciación social.

Estas funciones son las que permiten que las representaciones sociales ejerzan un rol en las prácticas y en las dinámicas de las relaciones sociales (Abric, 2001).

\subsection{REPRESENTACIONES SOCIALES EN LOS MEDIOS DE COMUNICACIÓN}

En su libro La psychanalyse, son image et son public, Moscovici describe las representaciones sociales del psicoanálisis presentes en la sociedad francesa de los años cincuenta, a partir del análisis de cuestionarios, aplicados a personas de distintos sectores de la población, y de artículos relacionados con el psicoanálisis, publicados en periódicos y revistas entre 1952 y 1956 (Farr, 1986). De esta forma, el estudio de las representaciones sociales ha estado ligado, desde sus orígenes, al análisis del discurso presente en los medios de comunicación escrita. En ese sentido, Farr (1986) plantea que la comunicación de masas refleja, crea y transforma las representaciones sociales, ordenando la forma y el contenido de las conversaciones, de forma tal que la transmisión de una gran cantidad de representaciones en los medios de comunicación es lo que las hace sociales. De acuerdo con Moscovici, "los medios masivos de comunicación, como tales, están desprovistos de eficacia al nivel del individuo. No modifican ni sus opiniones ni sus actitudes. Pero al penetrar en los grupos elementales de vecindad, familia, amigos, etcétera, mediante estos coloquios personales acaban por influir en él y por cambiarlos" (1993: 240). Esto significa que los sujetos, a partir de la información transmitida por los medios de comunicación, pueden interpretarla a base de su historia y a las interacciones con otros sujetos, y construir sus propias representaciones sociales respecto del tema en discusión (Rouquette, 1986).

Distintas investigaciones han analizado el discurso expresado en los medios de comunicación escrita para conocer las representaciones sociales que se transmiten por estos (e.g. Cerda y Opazo, 2013; Cuevas y Gutiérrez, 2011; Otero, 2011; Pardo, 2006). Cerda y Opazo (2013) analizaron 29 noticias publicadas en medios escritos para conocer las representaciones sociales acerca de la calidad de la educación presentes en el discurso de distintos actores, a raíz del conflicto estudiantil producido en Chile el 2011. A partir de su análisis, los autores identificaron tres categorías centrales que determinan las representaciones sociales acerca de la calidad de la educación que tienen los actores, como son: garantías de calidad, financiamiento y evaluación (Cerda y Opazo, 2013). Por su parte, Cuevas y Gutiérrez (2011) analizaron 30 artículos publicados en un periódico mexicano para estudiar las representaciones sociales que transmite el periódico acerca de la calidad académica de las universidades privadas. Los resultados dieron cuenta de dos 
representaciones sociales acerca de este tipo de instituciones: las universidades patito (de baja calidad) y las verdaderas universidades de paga, demostrando que para el periódico la calidad académica está relacionada con la originalidad de una marca comercial (Cuevas y Gutiérrez, 2011). Pardo (2006) analizó 350 noticias de cuatro periódicos colombianos para estudiar las representaciones sociales acerca de la impunidad en relación con poder público y el sistema jurídico. El análisis permitió identificar tres representaciones de la impunidad: como un hecho natural, como racionalidad, y como una lógica de producción. De acuerdo con la autora, la evolución de las representaciones sociales acerca de la impunidad está relacionada con transformaciones estructurales en el orden político, económico y social del país (Pardo, 2006).

\section{PROPUESTA DE INVESTIGACIÓN}

\subsection{PROBLEMA}

El 11 de agosto de 2011 se promulgó la Ley de Aseguramiento de la Calidad de la Educación Escolar (Ley No 20.529), esta corresponde a la cristalización del trabajo del Consejo Asesor Presidencial para la Calidad de la Educación, convocado por el gobierno de Michelle Bachelet como respuesta a las demandas realizadas por el movimiento estudiantil del 2006. Entre otros aspectos, esta ley creó la Agencia de Calidad de la Educación, cuyo objetivo es "evaluar y orientar el sistema educativo para que este propenda al mejoramiento de la calidad y equidad de las oportunidades educativas" (Ley $\mathrm{N}^{\mathrm{o}}$ 20.529). Para lograr este objetivo, la Agencia cumplirá cuatro funciones: a) evaluar los logros de aprendizaje de los alumnos, de acuerdo con el grado de cumplimiento de estándares, b) ordenar los establecimientos según los resultados de aprendizaje y otros indicadores de calidad educativa, c) realizar evaluaciones de desempeño a los establecimientos educacionales, directores y sostenedores, y d) proporcionar información a la comunidad (Agencia de Calidad de la Educación, 2013a; Ley No 20.529, 2011).

De estas funciones la que ha provocado más controversia es la ordenación de los establecimientos, la que clasificará las escuelas en cuatro categorías (Alto, Medio, MedioBajo e Insuficiente) y que, en el caso de aquellas clasificadas en la categoría Insuficiente por cuatro años seguidos, implicará, como última medida, la pérdida del reconocimiento oficial (Agencia de Calidad de la Educación, 2013a; Ley No 20.529, 2011). Es así como se ha generado un debate, a favor y en contra de la propuesta, en los medios de comunicación escritas, entre distintos actores del sistema educativo, principalmente a partir del 8 de abril de 2013, fecha en la que los Consejeros de la Agencia de Calidad de la Educación eligieron la metodología que permitirá realizar la clasificación de las escuelas (Agencia de Calidad de la Educación, 2013b). En el caso de los críticos, la discusión se ha centrado en los efectos negativos que tendría la propuesta tanto en las escuelas como en sus estudiantes; mientras que los defensores argumentan que este mecanismo permitirá mejorar la calidad de la educación.

El análisis del discurso, tanto de críticos como de defensores, permitirá conocer las representaciones sociales que los distintos actores del sistema educativo tienen respecto de la clasificación de escuelas. La importancia de estudiar este tema reside en que, de acuerdo con Moscovici (1993), los medios de comunicación influyen en 
los grupos sociales y pueden llegar a cambiar sus opiniones. De tal forma que los sujetos, a partir de la interpretación que realicen de la información transmitida por esta vía, pueden construir sus propias representaciones sociales respecto del tema en discusión (Rouquette, 1986).

\subsection{PREGUNTA E HIPÓTESIS DE INVESTIGACIÓN}

Sobre la base del problema planteado, la pregunta que orientará esta investigación es: ¿cuáles son las representaciones sociales de la clasificación de escuelas presentes en el discurso, en medios escritos, de distintos actores del sistema educativo? Al respecto se plantea la siguiente hipótesis exploratoria: los actores del sistema educativo tendrán representaciones sociales diferenciadas acerca de la clasificación de escuelas dependiendo de la posición, a favor o en contra, que adopten frente a este tema.

\subsection{OBJETO DE ESTUDIO}

De acuerdo a lo anterior, el objeto de investigación serán las representaciones sociales de la clasificación de escuelas presentes en el discurso, en medios escritos, de distintos actores del sistema educativo.

\subsection{OBJETIVO DE LA INVESTIGACIÓN}

El objetivo del presente trabajo es conocer las representaciones sociales de la clasificación de escuelas presentes en el discurso, en medios escritos, de distintos actores del sistema educativo.

\section{METODOLOGÍA DE INVESTIGACIÓN}

\subsection{TEORÍA FUNDAMENTADA}

La metodología que se utilizará para analizar los documentos seleccionados es la Teoría Fundamentada (Grounded Theory). Esta metodología de análisis, desarrollada por Glaser y Strauss en la década de 1960, da preferencia a los datos y al campo en estudio frente a los supuestos teóricos, estos no se deben aplicar al objeto que se investiga, sino que se "descubren" y formulan a partir de los datos empíricos (Flick, 2004). En este sentido, "el valor de la metodología radica en su capacidad, no solo de generar teoría, sino también de fundamentarla en los datos" (Strauss y Corbin, 2002: 9). El análisis de los datos se realiza por medio de la codificación, que corresponde a "representar las operaciones por las cuales los datos se desglosan, conceptualizan y vuelven a reunir de nuevas maneras" (Cit. en Flick, 2004: 193). La codificación se lleva a cabo en distintas etapas -abierta, axial, selectiva-, las que no necesariamente son secuenciales. En este sentido, la codificación es un proceso dinámico. El proceso básico de codificación es la codificación abierta, que es "el proceso analítico por medio del cual se identifican los conceptos" (Strauss y Corbin, 2002: 110). Los conceptos corresponden a "una representación abstracta de un acontecimiento, objeto o acción/ 
interacción que un investigador identifica como significativo en los datos" (Strauss y Corbin, 2002: 112). Aquellos conceptos que son nociones similares o se relacionan en el significado se agrupan bajo ideas más abstractas que se denominan categorías, las que a su vez representan fenómenos (Strauss y Corbin, 2002). Los fenómenos "son ideas analíticas pertinentes que emergen de nuestros datos (...) [y que] responden a la pregunta: ¿qué pasa aquí?” (Strauss y Corbin, 2002: 124). Una vez que se identifica una categoría se determinan sus propiedades y dimensiones (Strauss y Corbin, 2002). "Las propiedades son las características generales o específicas o los atributos de una categoría, [mientras que] las dimensiones representan la localización de una propiedad durante un continuo o rango" (Strauss y Corbin, 2002: 128). Posteriormente, en la codificación axial "las categorías se relacionan con sus subcategorías para formar unas explicaciones más precisas y completas sobre los fenómenos" (Strauss y Corbin, 2002: 135). Las subcategorías responden "preguntas sobre los fenómenos tales como cuándo, dónde, por qué, quién, cómo y con qué consecuencias" (Strauss y Corbin, 2002: 136). De acuerdo con Strauss y Corbin (2002), las relaciones entre las categorías y subcategorías se pueden organizar en un esquema que se denomina paradigma, cuyos componentes básicos son:

a) Condiciones: componen la estructura o conjunto de circunstancias o situaciones en las que están inscritos los fenómenos. Responden a preguntas de ¿por qué?, ¿dónde?, ¿cuándo?, ¿cómo?

b) Acciones/Interacciones: son las respuestas estratégicas o rutinarias dadas por los individuos o grupos a los asuntos, problemas o acontecimientos o sucesos que emergen bajo estas condiciones. Responden a las preguntas de ¿quién? y ¿cómo?

c) Consecuencias: son el resultado de las acciones e interacciones. Responden a preguntas como ¿qué sucede como resultado de estas acciones/interacciones?, ¿por qué los grupos no responden a situaciones por medio de acciones/interacciones?

A partir del establecimiento del paradigma es posible elaborar hipótesis que "corresponden a las intuiciones iniciales sobre la forma en que se relacionan los respecto de porque vinculan dos o más conceptos, explicando el por qué, el dónde y el cómo de un fenómeno" (Strauss y Corbin, 2002: 148).

\subsection{DESCRIPCIÓN DEL MATERIAL}

El material a analizar corresponde a columnas de opinión, cartas al director, editoriales, notas de prensa y blogs publicados tanto en diarios de circulación nacional (La Tercera, El Mercurio y La Segunda), en su versión impresa o digital, como en diarios digitales (El Mostrador y El Dínamo), así como también notas de prensa disponibles en sitios web de centros de investigación, facultades o departamentos de educación de universidades tradicionales, respecto del tema de la clasificación de escuelas. El número total de documentos recopilados fue 24 , los cuales se publicaron entre el 25 de marzo y el 3 de octubre de 2013, y corresponde a todo el material que se ha publicado hasta la fecha (principios de diciembre) acerca del tema. En la Tabla 1 se presentan las principales características de los documentos analizados. 
Tabla 1. Principales características de los documentos analizados

\begin{tabular}{|c|c|c|c|}
\hline $\begin{array}{c}\text { Fecha de } \\
\text { publicación }\end{array}$ & Tipo de material & $\begin{array}{c}\text { Medio de } \\
\text { comunicación }\end{array}$ & $\begin{array}{l}\text { Grado académico o profesión de el(los) } \\
\text { entrevistado(s) o autor(es) del documento }\end{array}$ \\
\hline 25-03-2013 & Columna de opinión & $\begin{array}{l}\text { Sitio web } \\
\text { Mide UC }\end{array}$ & - Magíster en Educación \\
\hline 09-04-2013 & Nota de prensa & La Segunda & - Magíster en Políticas Públicas \\
\hline 11-04-2013 & Editorial & La Segunda & \\
\hline 16-06-2013 & Editorial & La Tercera & \\
\hline $30-07-2013$ & Editorial & El Mercurio & \\
\hline 01-08-2013 & Columna de opinión & La Tercera & $\begin{array}{l}\text { - } \quad \text { Doctor en Educación } \\
\text { - } \quad \text { Doctor en Economía } \\
\text { - }\end{array}$ \\
\hline 06-08-2013 & Carta al director & La Tercera & - $\quad$ Master of Science and Education \\
\hline 08-08-2013 & Carta al director & La Tercera & - $\quad$ Ingeniero Comercial \\
\hline 11-08-2013 & Columna de opinión & La Tercera & - Socióloga \\
\hline 12-08-2013 & Carta al director & La Tercera & - $\quad$ Doctora en Ciencias de la Educación \\
\hline $12-08-2013$ & Nota de prensa & $\begin{array}{l}\text { Radio U. } \\
\text { de Chile }\end{array}$ & $\begin{array}{ll}- & \text { Sociólogo } \\
\text { - } & \text { Doctora en Sociología } \\
\text { - } & \text { Estudiante Liceo Municipal } \\
\end{array}$ \\
\hline 13-08-2013 & Carta al director & La Tercera & - Periodista \\
\hline 13-08-2013 & Carta abierta & $\begin{array}{l}\text { Sitio web } \\
\text { Educación } 2020\end{array}$ & \\
\hline $14-08-2013$ & Columna de opinión & La Tercera & $\begin{array}{ll}- & \text { Ingeniero Comercial } \\
- & \text { Ingeniero Comercial }\end{array}$ \\
\hline 14-08-2013 & $\begin{array}{l}\text { Carta al director } \\
\text { (El Mercurio, no } \\
\text { publicada) }\end{array}$ & $\begin{array}{l}\text { Sitio web } \\
\text { Facultad de } \\
\text { Educación, UC }\end{array}$ & $\begin{array}{ll} & \text { Ph.D., Sociólogo } \\
- & \text { Doctor en Educación } \\
- & \text { Doctor en Economía }\end{array}$ \\
\hline $15-08-2013$ & Carta al director & La Tercera & - Doctor en Educación \\
\hline $20-08-2013$ & Columna de opinión & Blog La Tercera & - $\quad$ Ingeniero Comercial \\
\hline $27-08-2013$ & Columna de opinión & La Tercera & - Ph.D. (c) en Economía \\
\hline 03-09-2013 & Columna de opinión & El Dínamo & - Sociólogo \\
\hline 03-09-2013 & Nota de prensa & $\begin{array}{l}\text { Sitio web CIDE, } \\
\text { UAH }\end{array}$ & - Master of Science and Education \\
\hline 22-09-2013 & Carta al director & El Mercurio & $\begin{array}{l}\text { - } \quad \text { Profesora de Inglés } \\
\text { - } \quad \text { Magíster en Políticas Sociales para Países } \\
\text { en Desarrollo } \\
\text { - } \quad \text { Magíster en Educación y Comunicación } \\
\text { - } \quad \text { Doctor en Ciencias Técnicas } \\
\text { - } \quad \text { Magíster en Sociología }\end{array}$ \\
\hline $25-09-2013$ & Editorial & El Mercurio & \\
\hline 03-10-2013 & Columna de opinión & El Mostrador & $\begin{array}{l}\text { - Ph.D. en Políticas Públicas y Ciencia } \\
\text { Política }\end{array}$ \\
\hline s.f. & Nota de prensa & $\begin{array}{l}\text { Sitio web Depto. } \\
\text { de Educación, } \\
\text { USACH }\end{array}$ & $\begin{array}{ll} & \text { Doctor en Ciencias de la Educación } \\
- & \text { Doctora en Sociología } \\
- & \text { Doctora (c) en Ciencias de la Educación }\end{array}$ \\
\hline
\end{tabular}




\subsection{ANÁLISIS DE LOS DOCUMENTOS}

La codificación abierta se realizó analizando los documentos oración por oración, preguntándose ¿cuál es la idea principal que tiene esta oración? (Strauss y Corbin, 2002), lo que permitió identificar 68 conceptos (códigos). Por medio del análisis comparativo, cada vez que se encontró una oración que compartía algunas características con otra ya codificada se le dio el mismo código; sin embargo, la mitad de los códigos (34 de 68) tiene asociado solo una oración (cita). Una vez identificados los conceptos, todos aquellos que eran similares, en términos conceptuales o de significado, se agruparon en categorías, y luego se procedió a identificar sus propiedades y dimensiones. Este proceso se vio dificultado porque los documentos analizados no abordan el tema de la clasificación de escuelas desde la misma perspectiva ni plantean los mismos elementos, por ello, si bien se podían identificar propiedades para las categorías construidas, no fue posible identificar distintas dimensiones para una misma propiedad que permitieran localizarla en un continuo o rango (Strauss y Corbin, 2002). Continuando con el análisis comparativo, en la codificación axial se reorganizaron las categorías construidas y se elaboraron cinco grandes familias de códigos. La Tabla 2 presenta la descripción de las familias de códigos construidas. Finalmente se trabajó en la elaboración del paradigma, el que muestra la relación entre las familias de códigos, identificando el contexto, el fenómeno, las condiciones, las acciones/interacciones y las consecuencias. El análisis de los documentos se realizó utilizando como herramienta el software ATLAS.ti versión 6.2.

Tabla 2. Descripción de las familias de códigos construidas

\begin{tabular}{|l|l|}
\hline \multicolumn{1}{|c|}{ Familia de códigos } & \multicolumn{1}{c|}{ Descripción } \\
\hline $\begin{array}{l}\text { Consecuencias de la } \\
\text { clasificación }\end{array}$ & $\begin{array}{l}\text { Alusiones a la Ley No 20.529 relacionadas con las consecuencias que } \\
\text { tendría la clasificación, como pérdida del reconocimiento oficial, visitas } \\
\text { evaluativas, entre otras. }\end{array}$ \\
\hline Efectos de la clasificación & $\begin{array}{l}\text { Opiniones relacionadas con los efectos a largo plazo que tendría la } \\
\text { clasificación, tanto en las escuelas como en sus estudiantes. }\end{array}$ \\
\hline Objetivos de la clasificación & $\begin{array}{l}\text { Menciones relacionadas con los objetivos de la clasificación y de la } \\
\text { Agencia de Calidad de la Educación. }\end{array}$ \\
\hline Opinión sobre la clasificación & Opiniones generales relacionadas con la clasificación de escuelas. \\
\hline $\begin{array}{l}\text { Opinión sobre la } \\
\text { metodología }\end{array}$ & $\begin{array}{l}\text { Opiniones generales relacionadas con la metodología de clasificación } \\
\text { de escuelas y, en específico, con sus indicadores. }\end{array}$ \\
\hline
\end{tabular}

\section{RESULTADOS}

La primera revisión de los documentos permitió identificar la existencia de tres posturas adoptadas por los distintos actores del sistema educativo frente al tema de la clasificación de escuelas. De esta forma, los documentos analizados se clasificaron en tres grupos: "defensores", "críticos", y "defensores con reparos". Estos últimos hacen referencia a aquellos documentos en los que se expresa una opinión a favor de la clasificación de escuelas, pero con algunas advertencias respecto de la metodología, indicadores, entre otros temas. Del total de documentos analizados, 11 corresponden a "defensores", diez a "críticos", y tres a "defensores con 
reparos". En el caso de los "defensores", cinco de ellos provienen de la Agencia de Calidad de la Educación, tres de la academia, y tres corresponden a Editoriales. Por su parte, los diez documentos "críticos" provienen del mundo de la academia y la investigación; mientras que los documentos clasificados como "defensores con reparos" corresponden a una Editorial y dos de provienen de la academia. Este estudio se centrará solo en los discursos de los "defensores" y los "críticos". En la Tabla 3 se presenta la distribución de los códigos identificados en el discurso de estos dos grupos, de acuerdo a la familia de códigos.

Tabla 3. Distribución de códigos (citas) por familia y grupo

\begin{tabular}{|l|c|c|}
\hline Grupo Familia de códigos & Defensores & Críticos \\
\hline Consecuencias de la clasificación & $6(25)$ & $3(7)$ \\
\hline Efectos de la clasificación & $2(3)$ & $8(16)$ \\
\hline Objetivos de la clasificación & $5(13)$ & $4(4)$ \\
\hline Opinión sobre la clasificación & $2(2)$ & $10(18)$ \\
\hline Opinión sobre la metodología & $12(17)$ & $19(28)$ \\
\hline Total de códigos (citas) & $27(60)$ & $44(73)$ \\
\hline
\end{tabular}

A continuación se procederá a analizar los discursos de cada postura. Primero se analizará la distribución de los códigos por familia, mostrando citas representativas del discurso, y luego se analizará la codificación axial.

\subsection{DEFENSORES}

\subsubsection{Análisis de la distribución de códigos}

De los códigos que se relacionan con las consecuencias que tendría la clasificación, los que concentran una mayor cantidad de citas son los que se refieren al asesoramiento y las visitas evaluativas que se realizarían a las escuelas categorizadas como Medio-bajo e Insuficiente.

"Pero desde el día uno, apenas sepamos qué colegios están en esa categoría, los visitaremos con expertos para determinar cómo se produce el aprendizaje dentro del aula para ver qué hay que corregir..."

"si bien todas las escuelas serán visitadas por la Agencia, los recursos y asesorías se focalizarán en aquellas que requieren un mayor apoyo"

Los dos códigos relacionados con los efectos de la clasificación se refieren al efecto positivo que esta tendría.

"Todas estas acciones, junto con el involucramiento de los padres, impulsará una educación equitativa y de calidad en el país"

Respecto de los códigos vinculados con los objetivos de la clasificación, el que concentra una mayor cantidad de citas es el que se refiere a la identificación de los establecimientos. 
"El fin de la ordenación no es 'rotular' establecimientos, sino identificar a los que presentan mayores necesidades"

"El objetivo de esta clasificación es identificar a los colegios que están teniendo un desempeño deficiente, medido a través de distintos indicadores de calidad"

En el caso de los dos códigos relacionados con opiniones generales relativas a la clasificación, estos hacen referencia a la clasificación como un mecanismo confiable y que considera el nivel de vulnerabilidad de los estudiantes.

“el foco de la agencia estará en los alumnos, así que se considerará la alta vulnerabilidad de los alumnos a la hora de categorizar las escuelas porque sabemos que esos estudiantes tienen un capital más bajo"

Los códigos que se relacionan con opiniones generales respecto de la metodología hacen referencia a los indicadores que se utilizarán o a la metodología misma.

"La metodología propuesta por la Agencia de Calidad lejos de ser diseñada apresuradamente entre cuatro paredes es fruto de un trabajo participativo de más de dos años"

"quisiera puntualizar que la ordenación incorpora considerablemente la vulnerabilidad y otras características de los estudiantes, por lo que no es correcto afirmar que ella "no da cuenta del capital sociocultural y económico como factor crítico de calidad'"

\subsubsection{Análisis de codificación axial}

A partir del proceso de codificación axial fue posible establecer que para los defensores, la clasificación de escuelas representa una herramienta orientadora para el funcionamiento de estas. En la Figura 1 se presenta el paradigma elaborado. La base de esta representación se encuentra en la visión positiva que este grupo tiene respecto del hecho de que una escuela sea categorizada como Medio-bajo e Insuficiente, haciendo referencia, por ejemplo, a las visitas evaluativas que se realizarían a este tipo de escuelas, y cómo este mecanismo ayudaría a mejorar la calidad de la educación.

Figura 1. Esquema representativo del discurso de los defensores de la clasificación de escuelas

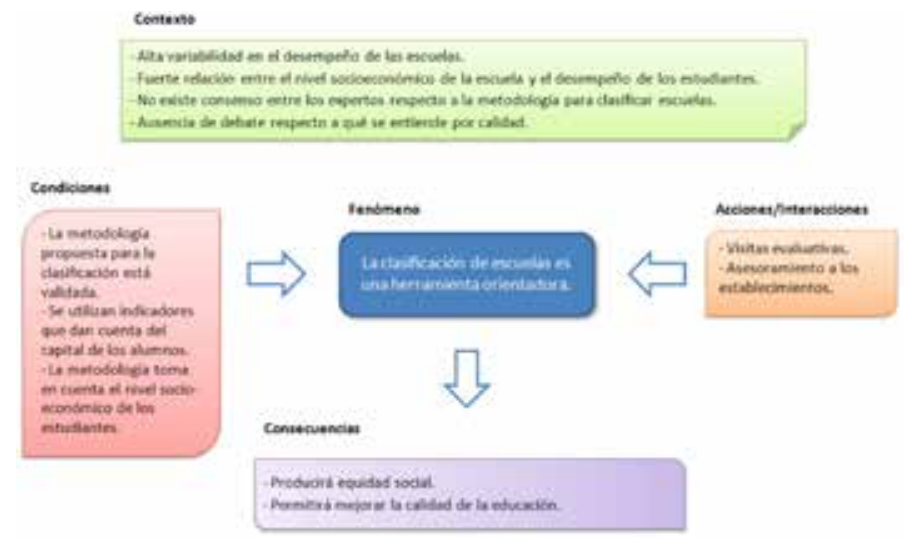




\subsection{CRÍTICOS}

\subsubsection{Análisis de la distribución de códigos}

Respecto de los códigos que se relacionan con las consecuencias de la clasificación de escuelas, el que concentra una mayor cantidad de citas es el que hace referencia al cierre de establecimientos.

"La aplicación de una clasificación de escuelas está alejada de ese propósito de mejoramiento y tiene grandes consecuencias. Las escuelas de nivel insuficiente serán cerradas. Si la metodología no es adecuada, se cerrarán escuelas equivocadamente"

"cada establecimiento queda con una calificación de Alto, Medio, Bajo e Insuficiente, arriesgándose a perder el reconocimiento oficial e incluso cerrar, si se mantiene en una misma categoría durante seis evaluaciones consecutivas"

De los códigos relacionados con los efectos de la clasificación, el que presenta una mayor cantidad de citas es el que se refiere a la estigmatización que este proceso produciría.

"Esta metodología de ordenación estigmatizará aún más a los niños y las escuelas vulnerables, sin ayudarles a mejorar"

"[la propuesta es] más de lo mismo, será una estigmatización más fina de los establecimientos, aunque la consideración de criterios intraescuela es sin duda un avance, pero aún insuficiente, dado que deberían considerarse otras variables tanto de estructura como de procesos escolares"

Los códigos vinculados con los objetivos de la clasificación hacen referencia, por ejemplo, a la identificación de establecimientos.

"La clasificación implicará la identificación de las escuelas que serán o no expuestas a distintos tipos de sanciones"

En relación con los códigos asociados con opiniones generales con respecto de la clasificación, el que concentra una mayor cantidad de citas es el que hace referencia al impacto que la esta tendría en el sistema escolar y en la calidad de la educación.

"[la medida de cerrar una escuela es] inadecuada, puesto que sería destruir una comunidad educativa y que provocaría consecuencias graves en el entorno social"

"Es una política que destruye la educación pública, y no tiene la intención de fomentar el buen desarrollo de las clases, ya que se cierran los colegios mal evaluados, que son principalmente municipales, que hoy tienen los peores resultado"

Respecto de los códigos que se relacionan con opiniones generales acerca de la metodología, los que concentran las citas son los que se refieren al peso del SIMCE y a la ausencia de debate relativo a qué metodología utilizar.

"Si el país ya cometió un grave error legislativo al otorgar un peso excesivo en la clasificación al Simce -instrumento profundamente cuestionado por su insuficiencia como indicador de calidad-" 
¿QQué debate público ha realizado el Mineduc para generar los acuerdos respecto de lo que entenderemos por buen desempeño de una escuela? ¿No es necesaria una discusión abierta con toda la comunidad, como se ha realizado en otros hitos de la reforma escolar?"

\subsubsection{Análisis de codificación axial}

A partir del proceso de codificación axial fue posible establecer que para los críticos, la clasificación de escuelas representa un mecanismo de castigo. En la Figura 2 se presenta el paradigma elaborado. La base de esta representación se encuentra en la visión negativa que este grupo tiene respecto de los efectos que la clasificación de escuelas produciría en los estudiantes, haciendo referencia, principalmente, a que los estudiantes de sectores vulnerables serían estigmatizados, y que aumentaría la segregación.

Figura 2. Esquema representativo del discurso de los defensores de la clasificación de escuelas

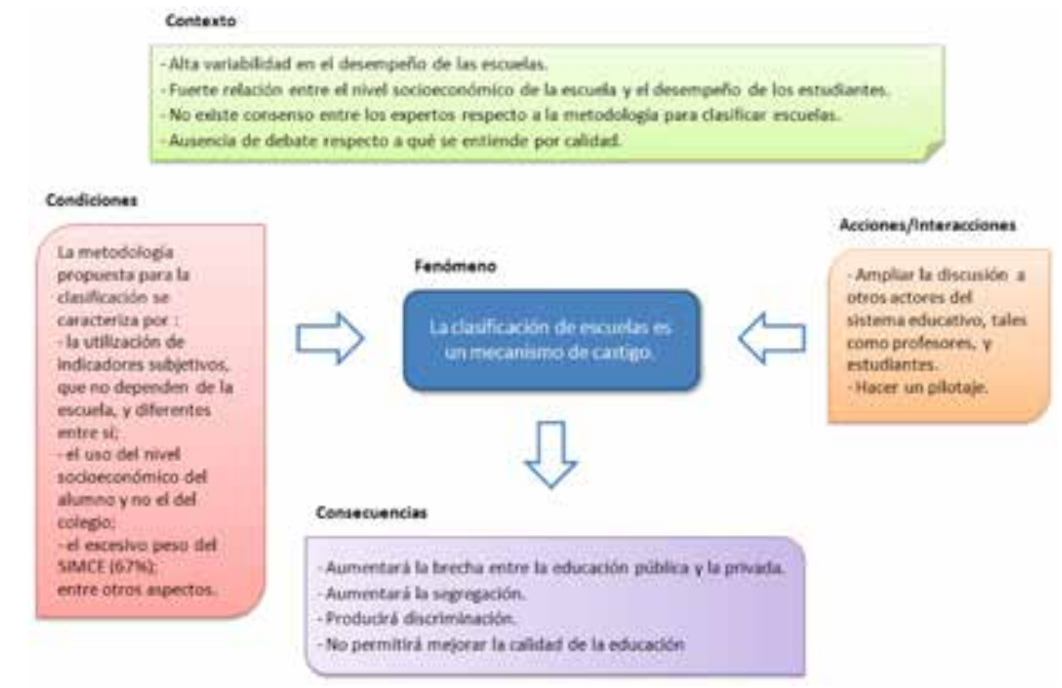

\section{DISCUSIÓN Y CONCLUSIONES}

En este trabajo se pretendió conocer las representaciones sociales de la clasificación de escuelas que tendrían distintos actores del sistema educativo, a partir del análisis de su discurso. Para ello se estudiaron documentos - cartas al director, columnas de opinión, editoriales, entre otros- publicados en distintos medios de comunicación escrita, impresos o digitales, aplicando como metodología de análisis la Teoría Fundamentada. Distintas investigaciones han analizado el discurso expresado en los medios de comunicación escrita para conocer las representaciones sociales acerca de, por ejemplo, la calidad de la educación (Cerda y Opazo, 2013), la calidad de las universidades privadas (Cuevas y Gutiérrez, 2011), la impunidad (Pardo, 2006), la salud de la población inmigrante (Otero, 2011). 
En relación con el material analizado, los documentos no abordaban el tema de la clasificación de escuelas desde la misma perspectiva ni planteaban los mismos elementos. Esto dificultó, por una parte, la identificación de conceptos que se relacionaran directamente con la pregunta de investigación planteada y, por otra, el encontrar citas que se vincularan con un mismo código, lo que se tradujo en que la mitad de los códigos (34 de 68) solo tienen asociado una cita. Por esta razón no se pudo obtener densidad conceptual ni demostrar el rango de variabilidad de las categorías como conceptos. Esto a su vez afectó el proceso de codificación axial, ya que no se pudo obtener suficiente material para todos los ejes del paradigma, principalmente para el eje Acciones/ Interacciones. Otra razón que podría explicar estas dificultades podría ser la extensión de los documentos analizados, medida en número de palabras, principalmente en el caso de las Cartas al Director, que variaban entre 200-300 palabras. Esto dificultaría que en el documento quedara plasmada en su totalidad la representación social del autor respecto del tema abordado. Algo parecido ocurriría con las Notas de Prensa, en ellas se tomó como discurso solo lo que decían textualmente los actores y no lo parafraseado por el periodista autor de la noticia, lo que redujo considerablemente el número de palabras. En este caso, el problema es que el discurso de los entrevistados queda reducido a extractos, en los que se expresa lo que es relevante para el periodista y no necesariamente para el entrevistado. Todo esto se traduce en que es más difícil encontrar, en el contenido de los documentos, elementos que permitan generar categorías y subcategorías que respondan las preguntas básicas de la teoría fundamentada: ¿cuándo?, ¿dónde?, ¿por qué?, ¿quién?, ¿cómo?, ¿con qué consecuencias?

Respecto del análisis realizado, es posible establecer que la hipótesis exploratoria planteada, relativa a que los actores del sistema educativo tendrían representaciones sociales diferenciadas acerca de la clasificación de escuelas dependiendo de la posición que adoptaran frente al tema, se relaciona con los paradigmas construidos. Para los defensores la clasificación de escuelas representa una herramienta orientadora, principalmente para las escuelas clasificadas en las dos categorías más bajas; mientras que para los críticos la clasificación representa un mecanismo de castigo, que presiona aún más a las escuelas vulnerables. Ante estas dos representaciones antagónicas, las consecuencias también son opuestas: para unos -los defensores- este mecanismo ayudaría a mejorar la calidad de la educación, que es finalmente la misión que le fue encomendada a la Agencia de Calidad; mientras que para los otros -los críticos- se produciría el efecto contrario, aumentando la segregación y la brecha entre la educación pública y privada. Sin embargo, en este punto es necesario recordar que casi la mitad ( 5 de 11) de las opiniones categorizadas como de defensa de la clasificación de escuelas proviene de personas relacionadas con la Agencia de Calidad, en respuesta a las críticas realizadas desde el mundo de la academia y la investigación, por lo que no resulta sorprendente la representación que estos tienen de la clasificación, ya que este sería un mecanismo que ellos mismos avalan o deben avalar.

A futuro, para estudios posteriores, sería interesante entrevistar a algunos de los autores de los documentos analizados, utilizando un guión diseñado con lógica de teoría fundamentada, para así poder obtener material que se pueda relacionar con los distintos componentes del paradigma. Esto permitiría profundizar en las representaciones sociales que los actores tienen respecto de la clasificación de escuelas y estudiar si esta representación varía al ampliar el discurso. Además, se podría entrevistar a otros actores, como directores, profesores, estudiantes, cuya opinión acerca de este tema no conocemos, y 
que posiblemente nos aportarían elementos relacionados con las estrategias para enfrentar el fenómeno. En este sentido, se podría plantear una hipótesis respecto de las relaciones que podrían existir entre las representaciones sociales y los tipos de actores, diferenciados entre "dentro del sistema" (e.g. directores, profesores, y estudiantes) y "fuera del sistema" (e.g. académicos, investigadores).

\section{REFERENCIAS BIBLIOGRÁFICAS}

Abric, J. C. (2001). Prácticas sociales y representaciones. México D.F.: Ediciones Coyoacán. Agencia de Calidad de la Educación (2013a). Balance de gestión integral año 2013. Recuperado el 28 de noviembre de 2013 desde http://www.agenciaeducacion.cl/

(2013b). Acta $N^{o} 32$ del Consejo de la Agencia de Calidad de la Educación. Recuperado el 28 de noviembre de 2013 desde http://www.agenciaeducacion.cl/wp-content/uploads/2013/02/ ACTA-N-32.pdf

Araya, S. (2002). Las representaciones sociales. Ejes teóricos para su discusión. San José: FLACSO.

Cerda, R. y Opazo, C. (2013). Las representaciones sociales de calidad educativa presente en los discursos en medios escritos: Un análisis en el contexto del conflicto estudiantil en Chile 2011. Estudios Pedagógicos, vol. 39 (1), 63-81.

Cuevas, Y. y Gutiérrez, S. (2011). La imagen de calidad de la educación superior privada en el diario El Universal. Reencuentro, (60), 58-67.

Dearden, L. \& Vignoles, A. (2011). Schools, markets and league tables. Fiscal Studies, vol. 32 (2), 179-186.

Durkheim, E. (2001). Las reglas del método sociológico. México D.F.: Fondo de Cultura Económica.

Duveen, G. y Lloyd, B. (2003). Las representaciones sociales como una perspectiva de la psicología social. En J. A. Castorina (Comp.), Representaciones sociales. Problemas teóricos y conocimientos infantiles (pp. 29-39). Barcelona: Gedisa.

El Mercurio (2013). Ranking: Los 100 colegios con mejor puntaje en SIMCE de $2^{o}$ básico. Recuperado el 3 de diciembre de 2013 desde http://www.emol.com/noticias/nacional/2013/06/20/604715/ranking-los-100-colegios-con-mejores-puntajes-en-el-simce-de-2-basico.html

Elstead, E. (2009). Schools which are named, shamed and blamed by the media: School accountability in Norway. Educational Assessment, Evaluation and Accountability, vol. 21 (2), 173-189.

Flick, U. (2004). Introducción a la investigación cualitativa. Madrid: Morata - Fundación Paideia.

Farr, R. M. (2003). De las representaciones colectivas a las representaciones sociales: Ida y vuelta. En J. A. Castorina (Comp.), Representaciones sociales. Problemas teóricos y conocimientos infantiles (pp. 153-175). Barcelona: Gedisa.

(1986). Las representaciones sociales. En S. Moscovici (Comp.), Psicología social II (pp. 495-506). Barcelona: Paidós.

Jodelet, D. (1986). La representación social: Fenómenos, concepto y teoría. En S. Moscovici (Comp.), Psicología social II (pp. 469-494). Barcelona: Paidós.

Karsten, S., Visscher, A. \& de Jong, T. (2001). Another side to the coin: The unintended effects of the publication of school performance data in England and France. Comparative Education, vol. 37 (2), 231-242.

Levačić, R. \& Woods, P. A. (2002). Raising school performance in the league tables (Part. 1): Disentangling the effects of social disadvantage. British Educational Research Journal, vol. 28 (2), 207-226. 
Ley $\mathrm{N}^{\circ} 20.529$ (2011). Sistema nacional de aseguramiento de la calidad de la educación parvularia, básica y media y su fiscalización. Recuperado el 25 de noviembre de 2013 desde http:// www.leychile.cl/Navegar?idNorma=1028635\&idVersion=2011-08-27

Machin, S. \& Vignoles, A. (2006). Education policy in the UK. London: Centre for the Economics of Education, London School of Economics.

Moscovici, S. (1993). La era de las multitudes. Un tratado histórico de la psicología de las masas. México D.F.: Fondo de Cultura Económica. (1979). El psicoanálisis, su imagen y su público. Buenos Aires: Huemul S.A.

Otero, L. (2011). Aproximación a las representaciones sociales sobre la salud de la población inmigrante en el discurso periodístico en prensa escrita española (2000-2006). Madrid: Escuela Nacional de Sanidad (ENS) - Instituto de Salud Carlos III - Ministerio de Ciencia e Innovación.

Pardo, N. (2006). Representaciones del discurso mediático: El caso de la impunidad en la prensa colombiana. Fronteiras, Estudos Midiáticos, vol. 8 (3), 241-254.

Petracci, M. y Kornblit, A. (2004). Representaciones sociales: Una teoría metodológicamente pluralista. En A. Kornblit (Ed.), Metodologías cualitativas en ciencias sociales (pp. 91-111). Buenos Aires: Biblos.

Power, S. \& Frandji, D. (2010). Education markets, the new politics of recognition and the increasing fatalism towards inequality. Journal of Education Policy, vol. 25 (3), 385-396.

Rouquette, M.-L. (1986). La comunicación de masas. En S. Moscovici (Comp.), Psicología social II (pp. 627-687). Barcelona: Paidós.

Strauss, A. y Corbin, J. (2002). Bases de la investigación cualitativa. Técnicas y procedimientos para desarrollar la teoría fundamentada. Medellín: Editorial Universidad de Antioquia.

West, A. \& Pennell, H. (2000). Publishing school examination results in England: Incentives and consequences. Educational Studies, vol. 26 (4), 423-436.

Woods, P. A. \& Levačić, R. (2002). Raising school performance in the league tables (Part. 2): Barriers to responsiveness in three disadvantaged schools. British Educational Research Journal, vol. 28 (2), 227-247. 
